\title{
Warped, Anisotropic Wormhole/Soliton Configurations in Vacuum 5D Gravity
}

\author{
Sergiu I. Vacaru * \\ Physics Department, CSU Fresno, Fresno, CA 93740-8031, USA, \\ and \\ Centro Multidisciplinar de Astrofisica - CENTRA, Departamento de Fisica, \\ Instituto Superior Tenico, Av. Rovisco Pais 1, Lisboa, 1049-001, Portugal \\ D. Singleton ${ }^{\dagger}$ \\ Physics Department, CSU Fresno, Fresno, CA 93740-8031, USA
}

(November 15, 2018)

\begin{abstract}
In this paper we apply the anholonomic frames method developed in refs. [1] - [1] to construct and study anisotropic vacuum field configurations in 5D gravity. Starting with an off-diagonal 5D metric, parameterized in terms of several ansatz functions, we show that using anholonomic frames greatly simplifies the resulting Einstein field equations. These simplified equations contain an interesting freedom in that one can chose one of the ansatz functions and then determine the remaining ansatz functions in terms of this choice. As examples we take one of the ansatz functions to be a solitonic solution of either the Kadomtsev-Petviashvili equation or the sine-Gordon equation. There are several interesting physical consequences of these solutions. First, a certain subclass of the solutions discussed in this paper have an exponential warp factor similar to that of the Randall-Sundrum model. However, the warp factor depends on more than just the $5^{\text {th }}$ coordinate. In addition the warp factor arises from anisotropic vacuum solution rather than from any explicit matter. Second, the solitonic character of these solutions might allow them to be interpreted either as gravitational models for particles (i.e. analogous to the' t Hooft-Polyakov monopole, but in the context of gravity), or as nonlinear, anisotropic gravitational waves.
\end{abstract}

PACS: 04.50.+h

\footnotetext{
*e-mails : sergiu_vacaru@yahoo.com, sergiuvacaru@venus.nipne.ro

†e-mail : dougs@csufresno.edu
} 


\section{INTRODUCTION}

The study of wormhole and black hole solutions in general relativity or its higher dimensional extensions usually starts with solutions with a high degree of symmetry [0], such as spherical symmetry. These highly symmetric solutions generally have metrics which are diagonal, and are thus easier to handle from a computational point of view. However, it is of interest to consider situations with less symmetry and metrics with off-diagonal components. Salam, Strathee and Percacci [6] have shown that including off-diagonal components in higher dimensional metrics is equivalent to including gauge fields. A specific example of this is given in refs. [7, 8] where 5D metrics with off-diagonal terms were considered, leading to solutions which were similar to spherically symmetric $4 \mathrm{D}$ wormhole or flux tube metrics but with "electric" and/or "magnetic" fields running along the throat of the wormhole. The "electromagnetic" fields arose as a consequence of the off-diagonal elements of the 5D metric.

Most realistic, physical situations do not have the high degree of symmetry that is found in the well known, exact solutions of general relativity. For example, it would be somewhat surprising if a pure Schwarzschild black hole existed (i.e. a black hole without angular momentum or charge). In order to study anisotropic configurations we use the method of anholonomic frames developed in [1, 2, (4) with associated nonlinear connections. The basic idea of this method is that the anholonomic frames can diagonalize metrics, which would have off-diagonal components in a holonomic coordinate frame. This substantially simplifies tensor computations but "elongates" the rule of partial differentiation and results in an anholonomic dynamics of particle/field interactions. Previously the anholonomic frames method was used [3] to construct anisotropic wormhole and flux tube solutions, which reduced to the solutions of [7 9] in the isotropic limit. These anisotropic solutions exhibited a variation or running of the "electromagnetic" charges as a result of the angular anisotropies and/or through variations of the extra spatial dimension. The solutions of ref. [3] were anisotropic deformations from spherically symmetric solutions. In ref. [10], we showed that the anholonomic frames method could be used to construct anisotropic wormhole and flux tube solutions to 5D Kaluza-Klein theory which possessed a host of different symmetries (elliptic, cylindrical, bipolar, toroidal).

In this paper we extend the results of [3, [10] to show that these locally anisotropic wormhole and flux tube solutions can be modified to include a class of metrics with a warped geometry with respect to the $5^{\text {th }}$ coordinate. In our approach the warp factor is not generated by some brane configuration with a special energy-momentum tensor as in the RandallSundrum (RS) scenario [11, but is induced by a specific anholonomic frame relation and nonlinear polarization of the vacuum 5D gravitational fields in the bulk. This supports the idea that off-diagonal metrics and anisotropies in extra dimensional vacuum gravity can model low dimensional interactions for both gravity and matter (more details can be found in [12] ). We will discuss the physical consequences of these solutions, in particular the gravitational running of the electromagnetic constants that occurs, and the anisotropic and solitonic nature of the electromagnetic and gravitational fields.

The paper is organized as follows: In section II we introduce an off-diagonal ansatz for $5 \mathrm{D}$ vacuum gravitational fields and outline the anholonomic frame method for this ansatz

by computing the nontrivial components of the Ricci tensor and constructing the general 
5D vacuum solutions for Einstein's equations. The coupled non-linear equations for the ansatz functions have a freedom that allows us to specify a particular form for one of the functions and then determine the other ansatz functions for this choice. We use this freedom to construct solutions that have one of the ansatz functions associated with 3D solitons. In section III we combine these solitonic solutions with certain 5D, "electric/magnetic" charged wormhole and flux tube solutions. The result is a vacuum solution with an anisotropic RS type warp factor. In section IV we show that it is possible to introduce an anisotropic deformation of these solutions so that the "electric" and "magnetic" charges of the solutions develop an anisotropic dependence. In section $\mathrm{V}$ we show that in addition to the spherical 3D hypersurface backgrounds that we used as a starting point for building the solitonically deformed wormhole and flux tube solutions, it is also possible to consider other 3D background geometries (ellipsoidal, toroidal, bipolar or cylindrical). Conclusions and physical consequences of the various solutions are given in section VI.

\section{A CLASS OF 5D VACUUM GRAVITATIONAL FIELDS}

In this section we outline the method of anholonomic frames with application to an ansatz for 5D vacuum gravitational metrics. These metrics have a mixture of holonomic and anholonomic variables, and are most naturally dealt with using anholonomic frames (the general theorems and corollaries are formulated and proven in refs. [4, 12]). We integrate the vacuum Einstein equations in explicit form and analyze the physical and mathematical properties of the 5D locally anisotropic vacuum solutions which can be reparametrized to yield warped and solitonic wormhole - flux tube configurations.

\section{A. Off-diagonal metric ansatz and anholonomic Einstein equations}

Let us consider a 5D pseudo-Riemannian spacetime of signature $(+,-,-,-,-)$ and denote the local coordinates $u^{\alpha}=\left(x^{i}, y^{a}\right)=\left(x^{1}=t, x^{2}=r, x^{3}=\theta, y^{4}=\chi, y^{5}=\varphi\right) . \chi$ is the $5^{\text {th }}$ coordinate, $t$ is the time like coordinate and $(r, \theta, \varphi)$ are the usual spherical spatial coordinates. The Greek indices are split into two subsets $x^{i}$ (holonomic coordinates) and $y^{a}$ (anholonomic coordinates) which are labeled respectively by Latin indices $i, j, k, \ldots=1,2,3$ and $a, b, \ldots=4,5$. The local coordinates will sometimes be written in the compact form $u=(x, y)$.

We begin our approach by considering a 5D quadratic line element

$$
d S^{2}=\Omega^{2}\left(x^{i}, \chi\right) g_{\alpha \beta}\left(x^{i}, \chi\right) d u^{\alpha} d u^{\beta}
$$

where $\Omega^{2}\left(x^{i}, \chi\right)$ is a conformal factor. The metric coefficients $g_{\alpha \beta}$ are parametrized by an off-diagonal matrix ansatz

$$
\left[\begin{array}{ccccc}
1+\left(w_{1}{ }^{2}+\zeta_{1}{ }^{2}\right) h_{4}+n_{1}{ }^{2} h_{5} & \left(w_{1} w_{2}+\zeta_{1} \zeta_{2}\right) h_{4}+n_{1} n_{2} h_{5} & \left(w_{1} w_{3}+\zeta_{1} \zeta_{3}\right) h_{4}+n_{1} n_{3} h_{5} & \left(w_{1}+\zeta_{1}\right) h_{4} & n_{1} h_{5} \\
\left(w_{1} w_{2}+\zeta_{1} \zeta_{2}\right) h_{4}+n_{1} n_{2} h_{5} & g_{2}+\left(w_{2}{ }^{2}+\zeta_{2}\right) h_{4}+n_{2} h_{5} & \left(w_{2} w_{3}++\zeta_{2} \zeta_{3}\right) h_{4}+n_{2} n_{3} h_{5} & \left(w_{2}+\zeta_{2}\right) h_{4} & n_{2} h_{5} \\
\left(w_{1} w_{3}+\zeta_{1} \zeta_{3}\right) h_{4}+n_{1} n_{3} h_{5} & \left(w_{2} w_{3}++\zeta_{2} \zeta_{3}\right) h_{4}+n_{2} n_{3} h_{5} & g_{3}+\left(w_{3}{ }^{2}+\zeta_{3}{ }^{2}\right) h_{4}+n_{3}{ }^{2} h_{5} & \left(w_{3}+\zeta_{3}\right) h_{4} & n_{3} h_{5} \\
\left(w_{1}+\zeta_{1}\right) h_{4} & \left(w_{2}+\zeta_{2}\right) h_{4} & \left(w_{3}+\zeta_{3}\right) h_{4} & h_{4} & 0 \\
n_{1} h_{5} & n_{2} h_{5} & n_{3} h_{5} & 0 & h_{5}
\end{array}\right]
$$

where the coefficients are smooth functions of the form: 
$g_{2,3}=g_{2,3}\left(x^{2}, x^{3}\right) \quad, \quad h_{4,5}=h_{4,5}\left(x^{i}, \chi\right), w_{i}=w_{i}\left(x^{i}, \chi\right), n_{i}=n_{i}\left(x^{i}, \chi\right), \quad \zeta_{i}=\zeta_{i}\left(x^{i}, \chi\right)$.

The quadratic line element (1) with metric coefficients (2) can be diagonalized,

$$
\delta S^{2}=\Omega^{2}\left[(d t)^{2}+g_{2}(d r)^{2}+g_{3}(d \theta)^{2}+h_{4}(\widehat{\delta} \chi)^{2}+h_{5}(\delta \varphi)^{2}\right]
$$

with respect to the anholonomic co-frame $\left(d x^{i}, \widehat{\delta} \chi, \delta \varphi\right)$, where

$$
\widehat{\delta} \chi=d \chi+\left(w_{i}+\zeta_{i}\right) d x^{i} \text { and } \delta \varphi=d \varphi+n_{i} d x^{i}
$$

which is dual to the frame $\left(\widehat{\delta}_{i}, \partial_{\chi}, \partial_{\varphi}\right)$, where

$$
\widehat{\delta}_{i}=\partial_{i}+\left(w_{i}+\zeta_{i}\right) \partial_{\chi}+n_{i} \partial_{\varphi}
$$

The various partial derivatives are abbreviated as $\dot{a}=\partial / \partial t, a^{\bullet}=\partial / \partial r, a^{\prime}=\partial / \partial \theta, a^{*}=$ $\partial / \partial \chi$. The first order anisotropy of metric (11) with ansatz (2) comes from the coefficients $w_{i}$ and $n_{i}$, while any second order anisotropy comes from having non-trivial coefficients $\zeta_{i}$ (details can be found in [4] and [2, 12]). For the trivial conformal factor $\Omega=1$ in (11) and (3) there is only a first order anisotropy parametrized by ansatz (2) with $\zeta_{i}=0$.

The nontrivial components of the $5 \mathrm{D}$ vacuum Einstein equations, $R_{\alpha}^{\beta}=0$, for the metric (3) given with respect to anholonomic frames (4) and (5) are:

$$
\begin{array}{r}
R_{2}^{2}=R_{3}^{3}=-\frac{1}{2 g_{2} g_{3}}\left[g_{3}^{\bullet \bullet}-\frac{g_{2}^{\bullet} g_{3}^{\bullet}}{2 g_{2}}-\frac{\left(g_{3}^{\bullet}\right)^{2}}{2 g_{3}}+g_{2}^{\prime \prime}-\frac{g_{2}^{\prime} g_{3}^{\prime}}{2 g_{3}}-\frac{\left(g_{2}^{\prime}\right)^{2}}{2 g_{2}}\right]=0, \\
R_{4}^{4}=R_{5}^{5}=-\frac{\beta}{2 h_{4} h_{5}}=0, \\
R_{4 i}=-w_{i} \frac{\beta}{2 h_{5}}-\frac{\alpha_{i}}{2 h_{5}}=0, \\
R_{5 i}=-\frac{h_{5}}{2 h_{4}}\left[n_{i}^{* *}+\gamma n_{i}^{*}\right]=0, \\
\partial_{i} \ln |\Omega|-\left(w_{i}+\zeta_{i}\right)(\ln |\Omega|)^{*}=0,
\end{array}
$$

where

$$
\begin{aligned}
\alpha_{i} & =\partial_{i} h_{5}^{*}-h_{5}^{*} \partial_{i} \ln \sqrt{\left|h_{4} h_{5}\right|}, \\
\beta & =h_{5}^{* *}-h_{5}^{*}\left[\ln \sqrt{\left.\left|h_{4} h_{5}\right|\right]^{*}},\right. \\
\gamma & =\frac{3 h_{5}^{*}}{2 h_{5}}-\frac{h_{4}^{*}}{h_{4}} .
\end{aligned}
$$

The anholonomic frames method has reduced the Einstein field equations for the complex, off-diagonal, 5D metric of (2) to a relatively "simple" system of coupled, nonlinear (6) - (10). In the following sections we will show that this simplification allows us to construct exact solutions to these $5 \mathrm{D}$ vacuum equations by embedding well known solitonic configurations into the system via the ansatz function $h_{4}, h_{5}$ or $n_{i}$. 


\section{B. Solutions of anholonomic vacuum Einstein equations}

The system of equations (6])-(10) has to some extent decoupled the equations for some of the ansatz functions through the use of anholonomic frames. Thus one has some freedom to chose one or more of the ansatz functions to take a particular, interesting form, and then solve for the remaining functions. For example one could chose $g_{2}(r, \theta)$ and then use equation (6) to solve for $g_{3}(r, \theta)$

- One solution of equation (6) is

$$
g_{1}(r, \theta)=g_{2}(r, \theta)=g(r, \theta)=g_{[0]}+g_{[1]} \exp \left[a_{2} r+a_{3} \theta\right]
$$

were $g_{[0]}, a_{2}$ and $a_{3}$ are some constants. This can be generalized by considering a coordinate transformation from $r, \theta \rightarrow \widetilde{x}^{2,3}(r, \theta)$ so that the $2 \mathrm{D}$ line element becomes conformally flat

$$
g_{2}(r, \theta)(d r)^{2}+g_{3}(r, \theta)(d \theta)^{2} \rightarrow g(r, \theta)\left[\left(d \widetilde{x}^{2}\right)^{2}+\epsilon\left(d \widetilde{x}^{3}\right)^{2}\right]
$$

this is always possible for $2 \mathrm{D}$ metrics. The explicit form of $\widetilde{x}^{2,3}$ depends on the signature $\epsilon= \pm 1$. With this transformation we write

$$
g_{1,2}(r, \theta)=g_{1,2[0]}+g_{1,2[1]} \exp \left[a_{2} \widetilde{x}^{2}(r, \theta)+a_{3} \widetilde{x}^{3}(r, \theta)\right]
$$

for constants $g_{1,2[0]}$ and $g_{1,2[1]}$. The simplest case for solutions to equation (6) is when the ansatz functions only depend on a single variable such as $g_{2}(r)$ and $g_{3}(\theta)$ so that the derivatives automatically vanish.

- Equation (7) relates two functions $h_{4}\left(x^{i}, \chi\right)$ and $h_{5}\left(x^{i}, \chi\right)$. There are two possibilities. First if $h_{4}\left(x^{i}, \chi\right)$ is given then $h_{5}\left(x^{i}, \chi\right)$ can be computed as

$$
\begin{aligned}
\sqrt{\left|h_{5}\right|} & =h_{5[0]}\left(x^{i}\right)+h_{5[1]}\left(x^{i}\right) \int \sqrt{\left|h_{4}\left(x^{i}, \chi\right)\right|} d \chi, \quad \text { for } h_{4}^{*}\left(x^{i}, \chi\right) \neq 0 \\
& =h_{5[0]}\left(x^{i}\right)+h_{5[1]}\left(x^{i}\right) \chi, \quad \text { for } h_{4}^{*}\left(x^{i}, \chi\right)=0
\end{aligned}
$$

where $h_{5[0,1]}\left(x^{i}\right)$ are functions determined by boundary conditions. Second, if $h_{5}\left(x^{i}, \chi\right)$ is given and $h_{5}^{*} \neq 0$ then $h_{4}\left(x^{i}, \chi\right)$ can be computed as

$$
\sqrt{\left|h_{4}\right|}=h_{[0]}\left(x^{i}\right)\left(\sqrt{\left|h_{5}\left(x^{i}, \chi\right)\right|}\right)^{*},
$$

with $h_{[0]}\left(x^{i}\right)$ given by boundary conditions.

- The exact solutions of (8) are

$$
w_{k}=\frac{\partial_{k} \ln \left[\frac{\sqrt{\left|h_{4} h_{5}\right|}}{\left|h_{5}^{*}\right|}\right]}{\partial_{\chi} \ln \left[\frac{\sqrt{\left|h_{4} h_{5}\right|}}{\left|h_{5}^{*}\right|}\right]},
$$


for $\partial_{\chi}=\partial / \partial \chi$ and $h_{5}^{*} \neq 0$. If $h_{5}^{*}=0$, or even $h_{5}^{*} \neq 0$ but $\beta=0$, the coefficients $w_{k}$ could be arbitrary functions of $\left(x^{i}, \chi\right)$. For vacuum Einstein equations this is a degenerate case which imposes the compatibility conditions $\beta=\alpha_{i}=0$, which are satisfied if the $h_{4}$ and $h_{5}$ are related via formula (13) with $h_{[0]}\left(x^{i}\right)=$ const. For simplicity, in this paper we will consider only vacuum configurations with $w_{k}=0$.

- The exact solution of (9) is

$$
\begin{aligned}
n_{k} & =n_{k[0]}\left(x^{i}\right)+n_{k[1]}\left(x^{i}\right) \int\left[\frac{h_{4}}{\left(\left|h_{5}\right|\right)^{3 / 2}}\right] d \chi, \quad \text { for } h_{5}^{*} \neq 0 ; \\
& =n_{k[0]}\left(x^{i}\right)+n_{k[1]}\left(x^{i}\right) \int h_{4} d \chi, \quad \text { for } h_{5}^{*}=0 ; \\
& =n_{k[0]}\left(x^{i}\right)+n_{k[1]}\left(x^{i}\right) \int\left[\frac{1}{\left(\sqrt{\left|h_{5}\right|}\right)^{3}}\right] d \chi \quad \text { for } h_{4}^{*}=0,
\end{aligned}
$$

where the functions $n_{k[0,1]}\left(x^{i}\right)$ are determined from boundary conditions.

- If $\partial_{i} \Omega=0$ and $\Omega^{*}=0$ the exact solution of (10) is given by an arbitrary function $\zeta_{i}=\zeta_{i}\left(x^{i}, \chi\right)$. If $\Omega^{*} \neq 0$ but $\partial_{i} \Omega=0$ we take $\zeta_{i}=0$. Finally if both $\Omega^{*} \neq 0$ and $\partial_{i} \Omega \neq 0, \zeta_{i}$ takes the form

$$
\zeta_{i}=\left(\Omega^{*}\right)^{-1} \partial_{i} \Omega
$$

$w_{i}$ does not show up in this solution since in this paper we are considering only the, $w_{i}=0$ case.

Because the metric coefficients $h_{4}$ and $h_{5}$ are solutions to equation (7) one has the freedom to define two new solutions as $\widehat{h}_{4}=\eta_{4} h_{4}, \widehat{h}_{5}=\eta_{5} h_{5}$ ] where $\eta_{4,5}$ could depend on $t, r, \theta$ or $\chi$. We call the functions $\eta_{4,5}=\eta_{4,5}(t, r, \theta, \chi)$ gravitational polarizations since they modify the behavior of the metric coefficients $h_{4}$ and $h_{5}$ in a manner similar to how a material modifies the behavior of electric and magnetic fields in media. The "renormalization" of $h_{4,5}$ into $\widehat{h}_{4,5}$ results in corresponding "renormalizations" $n_{i} \rightarrow \widehat{n}_{i}, \zeta_{i} \rightarrow \widehat{\zeta}_{i}$ and $\Omega \rightarrow \widehat{\Omega}$ which are to be computed from equations (12) - (16) with redefined coefficients (11), $\gamma \rightarrow \hat{\gamma}, \beta \rightarrow$ $\widehat{\beta}, \alpha_{i} \rightarrow \widehat{\alpha}_{i}$. We will use the freedom to renormalize $h_{4,5}$ via $\eta_{4,5}$ to modify certain electric and magnetically charged, 5D wormhole and flux tube solutions.

\section{3D solitonic configurations embedded in 5D gravity}

Vacuum gravitational 2D solitons in 4D Einstein gravity were originally studied by Belinski and Zakharov [21]. In refs. [1] it was shown that there are 3D solitons in 4D gravity and in anisotropic Taub-NUT backgrounds. Here we will show that it is possible to embed $3 \mathrm{D}$ solitonic configurations into the $5 \mathrm{D}$ gravitational system of the preceding section.

The simplest way to construct a solitonic, off-diagonal $5 \mathrm{D}$ vacuum metric is to take one of the ansatz coefficients $h_{4}, h_{5}$ or $n_{i}$ as a solitonic solution of some particular non-linear equation. Then carrying out the integrations of equations (12)-(16) yields all the remaining ansatz functions. In the next two subsections we analyze two explicit examples of such solitonic solutions. First we take either $h_{4}$ or $h_{5}$ as solitonic and then determine $n_{i}$ in terms of this choice. Second, we take one of the $n_{i}$ 's as solitonic and determine $h_{4}$ and $h_{5}$. 


\section{Solitonic $h_{4}$ or $h_{5}$}

The coefficient $h_{5}(r, \theta, \chi)$ can be required to be a solution of the Kadomtsev-Petviashvili $(\mathrm{KdP})$ equation [15] or the $(2+1)$ sine-Gordon (SG) equation. Methods of dealing with the $\mathrm{KdP}$ and other $2+1$ dimensional soliton equations can be found in refs. [16] and [17]. More detailed information on the SG equation can be found in 18 20. In the $\operatorname{KdP}$ case $h_{5}(r, \theta, \chi)$ satisfies the following equation

$$
h_{5}^{* *}+\epsilon\left(\dot{h}_{5}+6 h_{5} h_{5}^{\prime}+h_{5}^{\prime \prime \prime}\right)^{\prime}=0, \quad \epsilon= \pm 1,
$$

while in the SG case $h_{5}(r, \theta, \chi)$ satisfies

$$
h_{5}^{* *}+h_{5}^{\prime \prime}-\ddot{h}_{5}=\sin \left(h_{5}\right) .
$$

We will use the notation $h_{5}=h_{5}^{K P}$ or $h_{5}=h_{5}^{S G}$ depending on if $h_{5}$ satisfies equation (17), or (18) respectively.

Haven chosen a solitonic value of $h_{5}=h_{5}^{K P, S G}$, we can compute $h_{4}$ by applying equation (13),

$$
h_{4}=h_{4}^{K P, S G}=h_{[0]}^{2}\left[\left(\sqrt{h_{5}^{K P, S G}}\right)^{*}\right]^{2} .
$$

The $h_{4}$ determined in this way will not necessarily share the solitonic character of $h_{5}$. The next step is to substitute the values $h_{4}^{K P, S G}$ and $h_{5}^{K P, S G}$ into the equation (15) and find the respective values $n_{i}=n_{i}^{K P, S G}(r, \theta, \chi)$. This, up to some explicit integrations and differentiations, gives an exact solution of the $5 \mathrm{D}$ vacuum Einstein equations generated from a solitonic equation for $h_{5}$. For simplicity we will set $g_{2,3}=1$ so that the holonomic $2 \mathrm{D}$ background is trivial.

In this subsection we have generated solutions to the system of equations (6) -(10) by requiring that $h_{5}$ satisfy some solitonic equation. It is also possible to carry out a similar construction but with $h_{4}$ satisfying equation (17) or equation (18)).

\section{Solitonic $n_{i}$}

Next we consider the case when $n_{i}$ is the solitonic solution of either the KdP or the SG equation. Specially we will take $n_{3}$ as the solution of either the KdP or SG equation, setting the other two components to zero $\left(n_{1,2}=0\right)$.

For $n_{3}$ satisfying the KdP equations

$$
n_{3}^{* *}+\epsilon\left(n_{3}^{\bullet}+6 n_{3} n_{3}^{\prime}+n_{3}^{\prime \prime \prime}\right)^{\prime}=0, \quad \epsilon= \pm 1,
$$

in which case we write $n_{3}=n_{3}^{K P}$, while for $n_{3}$ satisfying the $\mathrm{SG}$ equation

$$
n_{3}^{* *}+n_{3}^{\prime \prime}-n_{3}^{\bullet \bullet}=\sin \left(n_{5}\right) \text {, }
$$

we write $n_{3}=n_{3}^{S G}$. We now want to find $h_{4}$ and $h_{5}$ for the given solitonic ansatz $n_{3}=n_{3}^{K P, S G}$. If $h_{5}^{*} \neq 0$ then $h_{4}$ can be expressed in terms of $h_{5}$ using formula (19) with $h_{[0]}=$ const. The coefficient $\gamma$ can be expressed in terms of $h_{5}$ from (11) 


$$
\gamma=\left(\ln \left[\frac{\left|h_{5}\right|^{3 / 2}}{\left(h_{5}^{*}\right)^{2}}\right]\right)^{*}
$$

Inserting (20) into equation (9) with the one nontrivial value $n_{3}=n_{3}^{K P, S G}$ we obtain an explicit equation for $h_{5}$

$$
h_{5}^{K P, S G}=\left[n_{[0]}\left(x^{i}\right) \int \sqrt{\left|n_{3}^{K P, S G}(r, \theta, \chi)\right|} d \chi+n_{[1]}\left(x^{i}\right)\right]^{4},
$$

were $n_{[0]}\left(x^{i}\right)$ and $n_{[1]}\left(x^{i}\right)$ are fixed from boundary conditions. Once the explicit form of $h_{5}$ is given via the above expression one can go back and obtain explicit expression for $h_{4}$ and $\gamma$ from equations (13) and (11).

The main conclusion of this subsection is that the ansatz (2) when treated with anholonomic frames has the freedom to pick one of the ansatz functions $\left(h_{4}, h_{5}\right.$, or $\left.n_{i}\right)$ to satisfy some 3D solitonic equation. Then in terms of this choice all the other ansatz functions can be generated up to carrying out some explicit integrations and differentiations. In this way it is possible to build exact solutions of the $5 \mathrm{D}$ vacuum Einstein equations with a solitonic character.

There are other variations of the above solitonic constructions that could be tried: instead of taking $h_{4,5}$ to depend on $r, \theta$ and $\chi$ we could carry out the construction with other dependences $\left(e . g . h_{4}(t, \theta, \chi), h_{5}(t, \theta, \chi)\right.$, or $h_{4}(t, r, \chi), h_{5}(t, r, \chi)$, or $\left.h_{4}(t, \chi), h_{5}(t, \chi)\right)$.

\section{WORMHOLE AND FLUX TUBE SOLUTIONS WITH A WARP FACTOR}

In this section we analyze a subclass of metrics (3) with a specific type of conformal factor parametrized as

$$
\Omega(t, r, \theta, \chi)=\Omega_{0}(t, r, \theta) e^{-k(t, r, \theta)|\chi|}
$$

The functions $k(t, r, \theta)$ and $\Omega_{0}(t, r, \theta)$ can be defined from boundary conditions or some desired limit. In this paper we will assume that $k(t, r, \theta)$ and $\Omega_{0}(t, r, \theta)$ are of the form of the radion or modulus field from brane and string cosmology 13 . In the original RS solution 111 the warp factor was of the form given in equation (21) but with $k(t, r, \theta)=$ const. This led to unstable solutions and less realistic cosmological scenarios. Some of these problems could be avoided by allowing the constant in the exponential warp factor to become a variable. A consequence of this was the introduction of the radion or modulus field. More details of this can be found in refs. [13] where different forms of the radion field, $k(t, r, \theta)$, were proposed to generate anisotropic warped compactifications or inflationary scenarios, which are compatible with string gravity or other quantum gravity models.

From equation (16) the parametrization in equation (21) results in a second order anisotropy

$$
\zeta_{i}=\left(\Omega^{*}\right)^{-1} \partial_{i} \Omega=-k_{\varepsilon}^{-1}(t, r, \theta) \partial_{i} \ln \left|\Omega_{0}\right|+|\chi| \partial_{i} \ln \left|k_{\varepsilon}(t, r, \theta)\right|
$$

$k_{\varepsilon}=k$, if $\chi>0$ and $k_{\varepsilon}=-k$, if $\chi<0$. These $\zeta_{i}$ 's can then be used to calculate the anholonomically "elongated" partial derivatives in (4) and (5). 
For simplicity, in this paper we shall deal only with exponential conformal factors of the form

$$
\Omega_{0} \simeq 1, k \simeq k(t)
$$

with a linear dependence on the $5^{\text {th }}$ coordinate $\chi$

$$
\zeta_{1}(t, \chi)=|\chi| \partial_{t} \ln |k(t)|, \zeta_{2}=\zeta_{3}=0
$$

Putting these requirements together leads to a metric of the form

$$
\begin{aligned}
\delta S^{2}= & e^{-2 k(t)|\chi|}\left[(d t)^{2}+g_{2}(r, \theta)(d r)^{2}+g_{3}(r, \theta)(d \theta)^{2}\right. \\
& \left.+\eta_{4}(t, r, \theta, \chi) h_{4}(r, \theta)(\widehat{\delta} \chi)^{2}+\eta_{5}(t, r, \theta, \chi) h_{5}(r, \theta)(\delta \varphi)^{2}\right] .
\end{aligned}
$$

where we have inserted the gravitational polarizations, $\eta_{4,5}$. Such a metric with its radion field warp factor is studied in connection with brane and string cosmological models. The new feature in the solutions that we construct is that they are vacuum solutions which do not have any explicit matter.

In the following subsections we will show how the isotropic wormhole/flux tube solutions of ref. [8,9] can be deformed into the above anisotropic form with a warp factor and solitonic background.

\section{A. 5D isotropic wormholes and anisotropic solitons}

We give a brief review of the locally isotropic wormhole and flux tube solutions constructed in refs. [8,9] (DS-solutions). Then in stages we modify these locally isotropic solutions to incorporate the solitonic ansatz function and the radion like warp factor.

\section{The locally isotropic DS-solutions}

The following form for the ansatz functions in ansatz (23)

$$
\begin{aligned}
& g_{1}=1, \quad g_{2}=-1, \quad g_{3}=-a(r), \quad k=k_{t}=k_{r}=k_{\theta}=0, \\
& h_{4}=-a(r) \sin ^{2} \theta, \quad h_{5}=-r_{0}^{2} e^{2 \psi(r)}, \quad \eta_{4}=\eta_{5}=1, \\
& n_{1}=\omega(r), \quad n_{2}=0, \quad n_{3}=n_{[0]} \cos \theta
\end{aligned}
$$

defines a trivial, locally isotropic solution of the vacuum Einstein equations (7)-(9) which satisfies the conditions $h_{4,5}^{*}=0$. The analytic or numerical determined forms for $a(r), \omega(r)$ and $\psi(r)$ are given in refs. 8,93. Inserting the functions from equation (24) into the ansatz (23) gives

$$
d S_{(D S)}^{2}=d t^{2}-d r^{2}-a(r)\left(d \theta^{2}+\sin ^{2} \theta d \varphi^{2}\right)-r_{0}^{2} e^{2 \psi(r)}\left[d \chi+\omega(r) d t+n_{[0]} \cos \theta d \theta\right]^{2}
$$

Up to a conformal factor and a rotation of the $5^{\text {th }}$ coordinate this is equivalent to the DS-solution for 5D wormhole/ flux tube configurations 8,9] (more details can be found in refs. [3], or [10]). In (25) $n_{[0]}$ is taken to be an integer; $r \in\left\{-R_{0},+R_{0}\right\}\left(R_{0} \leq \infty\right)$ 
and $r_{0}$ is a constant. All functions $\psi(r)$ and $a(r)$ were taken to be even functions of $r$ satisfying $\psi^{\prime}(0)=a^{\prime}(0)=0$. The coefficient $\omega(r)$ in (25) is treated as the $t$-component of the electromagnetic potential and $n_{[0]} \cos \theta$ as the $\theta$-component. These electromagnetic potentials lead to the metric having radial Kaluza-Klein "electrical" and "magnetic" fields. The 5D Kaluza-Klein "electric" field is

$$
E_{K K}=r_{0} \omega^{\prime} e^{3 \psi}=\frac{q_{0}}{a(r)}
$$

the "electric" charge $q_{0}=r_{0} \omega^{\prime}(0)$ can be parametrized as $q_{0}=2 \sqrt{a(0)} \sin \alpha_{0}$. The corresponding dual, "magnetic" field is

$$
H_{K K}=\frac{Q_{0}}{a(r)}
$$

with "magnetic" charge $Q_{0}=n r_{0}$ parametrized as $Q_{0}=2 \sqrt{a(0)} \cos \alpha_{0}$, The following circle relation

$$
\frac{\left(q_{0}^{2}+Q_{0}^{2}\right)}{4 a(0)}=1
$$

relates the "electric" and "magnetic" charges. This relation between the "electric" and "magnetic" charges will still remain valid even when these charges are generalized so as to have an anisotropic dependence on the coordinates. In this case equation (28) fixes the behavior of one charge in terms of the other. For different relative values of the "electric" and "magnetic" charges it was found that the solution changed from a wormhole (when the "electric" charged was larger than the "magnetic" charge) to a flux tube (when the "magnetic" charge was equal to or larger than the "electric" charge).

\section{Warped, anisotropic solitonic extension of the DS-solution}

The simplest way to obtain anisotropic wormhole / flux tube solutions [3] is to take $r_{0}^{2}$ from (25) not as a constant, but as "renormalized" via $r_{0}^{2} \rightarrow \widehat{r}_{0}^{2}=\widehat{r}_{0}^{2}(r, \theta, v)$, where the anisotropic coordinate, $v$ could be $\chi$ or $\varphi$ (i.e. we have two classes of anisotropic solutions, one with the $5^{\text {th }}$ coordinate and another with the angular coordinate $\varphi$ ). A number of anisotropic wormhole/ flux tube solutions in vacuum 5D gravity, for both cases - anisotropies in $\chi$ or $\varphi$ - were constructed and analyzed in refs. [3, 10]. For simplicity, in this paper we restrict our considerations only to warp factors, anisotropies with respect to the extra dimensional coordinate, and possible solitonic polarizations with respect to the variables $(t, r, \theta, \chi)$. Anisotropies on sets of coordinates like $(t, r, \theta, \varphi)$ can be defined in a similar fashion and we omit such considerations.

From the isotropic solution (24) we generated [3, 10] an anisotropic solution by taking

$$
\widehat{h}_{4}(r, \theta)=h_{4}(r, \theta)=-a(r) \sin ^{2} \theta,
$$

with $\eta_{4}=1$ so that $\widehat{h}_{4}^{*}=h_{4}^{*}=0$, but $\widehat{h}_{5}^{*}=\eta_{5}^{*}(r, \theta, \chi) h_{5}(r) \neq 0$. We parametrized

$$
\widehat{r}_{0}^{2}(r, \theta, \chi) \simeq r_{0(0)}^{2}[1+\varpi(r, \theta) \chi]^{2}
$$


so that

$$
\widehat{h}_{5}(r, \theta, \chi)=\eta_{5}(r, \theta, \chi) h_{5}(r), \quad h_{5}(r)=-r_{0}^{2} e^{2 \psi(r)}, \quad \eta_{5}(r, \theta, \chi)=[1+\varpi(r, \theta) \chi]^{2} .
$$

The term $\varpi(r, \theta) \chi$ parameterizes the dependence of $\widehat{r}_{0}^{2}$ on $r, \theta, \chi$ In [3] we took the simple case of $\varpi(r, \theta)=$ const. in order to illustrate that the DS-solutions admit a running of the "electromagnetic" constant with respect to the $5^{\text {th }}$ coordinate $\chi$. In general, the physical constants could also be anisotropically polarized through the holonomic coordinates $(r, \theta)$, which is accomplished by letting $\varpi=$ const. $\rightarrow \varpi(r, \theta)$.

The ansatz functions $n_{2,3}$ depend on the anisotropic variable $\chi$ in the following way

$$
n_{3}(r, \theta, \chi)=n_{3[0]}(r, \theta)+n_{3[1]}(r, \theta)[1+\varpi(r, \theta) \chi]^{-2} .
$$

In the locally isotropic limit $(\varpi \chi \rightarrow 0)$ one recovers the form of equation (25) with $n_{2[0,1]}=0$ , $n_{3[0]}=0, n_{3[1]}(r, \theta)=n \cos \theta$ and $n_{1}=\omega(r)$.

The $5 \mathrm{D}$ gravitational vacuum polarization induced by variation of the "constant" $\widehat{r}_{0}(\chi)$ renormalizes the electromagnetic charge as $q(\theta, \chi)=\widehat{r}_{0}(r=0, \theta, \chi) \omega^{\prime}(r=0)$. In terms of the angular parametrization the "electric" charge becomes

$$
q(\theta, \chi)=2 \sqrt{a(0)} \sin \alpha(\theta, \chi),
$$

and the "electric" field from (26) becomes

$$
E_{K K}=\frac{q(\theta, \chi)}{a(r)}
$$

The renormalization of the magnetic charge, $Q_{0} \rightarrow Q(\theta, \chi)$, can be obtained using the renormalized "electric" charge in relationship (28) and solving for $Q(\theta, \chi)$. The form of (28) implies that the running of $Q(\theta, \chi)$ will be the opposite that of $q(\theta, \chi)$. For example, if $q(\theta, \chi)$ increases with $\chi$ then $Q(\theta, \chi)$ will decrease. The locally anisotropic polarizations $\alpha(\theta, \chi)$ are either defined from experimental data or computed from a quantum model of 5D gravity.

Thus the following ansatz functions for (23)

$$
\begin{aligned}
& g_{1}=1, \quad g_{2}=-1, \quad g_{3}=-a(r), \quad k=k_{t}=k_{r}=k_{\theta}=0, \\
& \widehat{h}_{4}=h_{4}=-a(r) \sin ^{2} \theta, \quad \eta_{4}=1 \\
& \widehat{h}_{5}=\eta_{5} h_{5}, \quad h_{5}(r)=-r_{0}^{2} e^{2 \psi(r)}, \quad \eta_{5}=[1+\varpi(r, \theta) \chi]^{2} \\
& n_{1}=\omega(r), \quad n_{2}=0, \quad n_{3}=n_{[0]} \cos \theta[1+\varpi(r, \theta) \chi]^{-2},
\end{aligned}
$$

generates an anisotropic wormhole / flux tube metric whose electromagnetic charges have become dependent on $(r, \theta, \chi)$.

Next we further modify the locally anisotropic configuration in equation (30) to include a warp factor and a solitonic deformation. Instead of $\widehat{h}_{5}$ from (30) we choose

$$
\widehat{h}_{5}=h_{5}^{[K P, S G]}(r, \theta, \chi)=\eta_{5}^{[K P, S G]}(r, \theta, \chi) \eta_{5}(r, \theta) h_{5}(r), \quad h_{5}(r)=-r_{0}^{2} e^{2 \psi(r)},
$$

where $\eta_{5}^{[K P, S G]}(r, \theta, \chi)$ is defined by the requirement that $\widehat{h}_{5}$ be a solution of the KdP equation (17), or of the SG equation (18). Then we modify $\widehat{h}_{4}$ as 


$$
\widehat{h}_{4}(r, \theta, \chi)=\eta_{4}^{[K P, S G]}(r, \theta, \chi) h_{4}(r, \theta),
$$

where $h_{4}=-a(r) \sin ^{2} \theta$ as in ([30). Remembering that $h_{4}(r, \theta)=h_{[0]}^{2} \eta_{5}(r, \theta) h_{5}(r)$ with $h_{[0]}=$ const, then gives $\eta_{4}^{[K P, S G]}(r, \theta, \chi)$ from equation (19) as

$$
\eta_{4}^{K P, S G}=\left[\left(\sqrt{\eta_{5}^{K P, S G}}\right)^{*}\right]^{2} .
$$

The values of $n_{i}=n_{i}^{[K P, S G]}$ are computed by introducing $\widehat{h}_{4}$ and $\widehat{h}_{5}$ into the formulas (15). Next we require that $n_{i[0,1]}\left(x_{i}\right)$ satisfy boundary conditions so as to give nontrivial values for $n_{1}^{[K P]}=\omega(r)$ and $n_{3}=n_{[0]} \cos \theta \mu^{[K P, S G]}(r, \theta, \chi)$, where $\mu^{[K P, S G]}(r, \theta, \chi)$ can be picked out after performing the $\chi$ integration in equation (15) for the functions from equations (31) and (32).

Finally we introduce a conformal factor (22). This results in a constant shift of the second order anisotropy $\zeta_{1}(t, \chi)=|\chi| \partial_{t} \ln |k(t)|, \zeta_{2}=\zeta_{3}=0$. Collecting all the results together we see that

$$
\begin{aligned}
& g_{1}=1, \quad g_{2}=-1, \quad g_{3}=-a(r), \quad \Omega=e^{-k(t)|\chi|}, \\
& \widehat{h}_{4}=\eta_{4} h_{4}, \quad h_{4}=-a(r) \sin ^{2} \theta, \quad \eta_{4}^{K P, S G}=\left[\left(\sqrt{\eta_{5}^{K P, S G}}\right)^{*}\right]^{2}, \\
& \widehat{h}_{5}=\eta_{5}^{[K P, S G]}(r, \theta, \chi) \eta_{5}(r, \theta) h_{5}, \quad h_{5}(r)=-r_{0}^{2} e^{2 \psi(r)}, \quad h_{4}(r, \theta)=h_{[0]}^{2} \eta_{5}(r, \theta) h_{5}(r), \\
& \zeta_{1}=|\chi| \partial_{t} \ln |k(t)|, \quad n_{1}=\omega(r), \quad n_{2}=0, \quad n_{3}=n_{[0]} \cos \theta \mu^{[K P, S G]}(r, \theta, \chi),
\end{aligned}
$$

gives a new, exact solution of the $5 \mathrm{D}$ vacuum Einstein equations. It has an exponential warp factor in the extra dimensional coordinate, and is also time dependent. The isotropic wormhole / flux tube configuration of ref. 9] has been self-consistently embedded on an anisotropic, KdP or SG solitonic gravitational background. The "electromagnetic" constants of the solutions are anisotropically renormalized and scale with respect to the $5^{\text {th }}$ coordinate. The Kaluza-Klein magnetic field component exhibits a 3D solitonic polarization.

The final remark in this section is that we can also consider 3D solitons of the KdP or SG equations with a time like dependence in the polarization functions like $\eta_{4,5}^{[K P, S G]}(t, \theta, \chi)$. In this case the solution would describe a warped anisotropic wormhole / flux tube configuration moving self-consistently in an effective 4D spacetime. Such a configuration could be thought of as a traveling gravitational soliton which might be searched for in a gravitational wave detector such as LIGO.

\section{GRAVITATIONAL POLARIZATION OF KALUZA-KLEIN CHARGES}

We can further generalize the form (30) and (33) to generate new solutions of the 5D vacuum Einstein equations with deformations of the constants $r_{0}^{2}$ and $n_{[0]}$ with respect to the $\theta$ variable. These $\theta$ deformations take the form of the equation for an ellipsoid in polar coordinates. This again leads to renormalization of electric $(q)$ and magnetic $(Q)$ charges.

\section{A. Gravitational renormalization of Kaluza-Klein charges via variable $r_{0}$}

In this subsection we give a solution for which the Kaluza-Klein charges are gravitationally renormalized by the radius becoming dependent on $\theta$ (i.e. in equation (28) $a(0) \rightarrow a(\theta)$. 
The easiest way to obtain such $\theta$-polarizations for the solutions of (29) is to recast the ansatz functions (33) as $\widehat{h}_{5}=\bar{\eta}_{5} h_{5}(r)$ with

$$
\bar{\eta}_{5}=\left[1+\varepsilon_{r} \cos \theta\right]^{-2} \eta_{5}^{[K P, S G]}(r, \theta, \chi) \eta_{5}(r, \theta),
$$

and

$$
\widehat{r}_{0}^{2}(r, \theta, \chi) \simeq r_{0(0)}^{2}\left[1+\varepsilon_{r} \cos \theta\right]^{-2} \eta_{5}(r, \theta) \eta_{5}^{[K P, S G]}(r, \theta, \chi)
$$

where $\varepsilon_{r}$ is the eccentricity of an ellipse. The "constant", $\widehat{r}_{0}$, has both an elliptic variation in $\theta$ (i.e. $\left.r_{0(0)}\left[1+\varepsilon_{r} \cos \theta\right]^{-1}\right)$ and a solitonic variation with respect to the $5^{\text {th }}$ coordinate, $\chi$, and the variables $r, \theta$.

With these solutions the 5D Kaluza-Klein charges get renormalized through the elliptic variation of $\widehat{r}_{0}\left(r, \theta, \varepsilon_{r}, \chi\right)$, and the "electric" charge becomes

$$
q(r, \theta, \chi)=r_{0} \sqrt{\bar{\eta}_{5}\left(r, \theta, \varepsilon_{r}, \chi\right)} \omega^{\prime}(0)=\sqrt{\bar{\eta}_{5}\left(r, \theta, \varepsilon_{r}, \chi\right)} q_{0}
$$

In terms of the angular parametrization we find

$$
q(r, \theta, \chi)=2 \sqrt{a(0)} \sqrt{\bar{\eta}_{5}\left(r, \theta, \varepsilon_{r}, \chi\right)} \sin \alpha_{0},
$$

The "electric" field (26) transforms into

$$
E_{K K}=\frac{q(r, \theta, \chi)}{a(r)}=\frac{q_{0}}{\left(\sqrt{\bar{\eta}_{5}}\right)^{-1} a(r)}
$$

$\left(\sqrt{\bar{\eta}_{5}}\right)^{-1}$ can be treated as an anisotropic, gravitationally induced permittivity. The renormalization of the magnetic charge, $Q_{0} \rightarrow Q(r, \theta, \chi)$, can be obtained from equation (28) using $q(r, \theta, \chi)$ from above. In this case the corresponding dual, "magnetic" field is $H_{K K}=Q(r, \theta, \chi) / a(r)$ with the "magnetic" charge $Q_{0}=n_{[0]} r_{0}$ given by

$$
Q=2 \sqrt{a(0)} \sqrt{\bar{\eta}_{5}\left(r, \theta, \varepsilon_{r}, \chi\right)} \cos \alpha_{0}
$$

These gravitationally polarized charges satisfy the circumference equation (28) with variable radius $2 \sqrt{a(0)} \sqrt{\bar{\eta}_{5}\left(r, \theta, \varepsilon_{r}, \chi\right)}$

$$
\frac{\left(q_{0}^{2}+Q_{0}^{2}\right)}{4 a(0) \bar{\eta}_{5}\left(r, \theta, \varepsilon_{r}, \chi\right)}=1 \text {. }
$$

The new result - as compared with the elliptically polarized solutions of [10] - is that we have an additional dependence on the radial coordinate $r$. Also the anisotropic, gravitationally induced permittivity, $\left(\sqrt{\bar{\eta}_{5}}\right)^{-1}$, arises in connection with the 3D KdP or SG soliton solutions which have been embedded in the 5D Einstein equations. 


\section{B. Gravitational renormalization of Kaluza-Klein charges via $r_{0}$ and $n$}

A different class of solutions from those given in equations (30) and (33) can be constructed if, in addition to $r_{0}$, we allow the $n_{[0]}$ in the $n_{[0]} \cos \theta$ term from equation (25) to vary. For solutions with anisotropic extra dimensional coordinate, this variable $n_{[0]}$ will affect $n_{3}$. The variability of $r_{0}$ and $n_{[0]}$ is parameterized using the gravitational vacuum polarizations $\kappa_{r}(r, \theta, \chi)$ and $\kappa_{n}(r, \theta, \chi)$ as

$$
r_{0} \rightarrow \widehat{r}_{0}=\frac{r_{0}}{\kappa_{r}(r, \theta, \chi)} \quad \text { and } \quad n_{3} \rightarrow \widehat{n}_{3}=\frac{n_{[0]}}{\kappa_{n}(r, \theta, \chi)}
$$

where

$$
\kappa_{r}(r, \theta, \chi)=\left[\sqrt{\eta_{4}(r, \theta, \chi)}\right]^{-1} \quad \text { or } \quad=\left[\sqrt{\eta_{5}(r, \theta, \chi)}\right]^{-1} .
$$

The polarized charges are

$$
q=\frac{q_{0}}{\kappa_{r}}=\frac{2 \sqrt{a(0)} \sin \alpha_{0}}{\kappa_{r}}
$$

and

$$
Q=\frac{Q_{0}}{\kappa_{n}}=\frac{2 \sqrt{a(0)} \cos \alpha_{0}}{\kappa_{n}},
$$

Using these charges in equation (28) gives the formula for an ellipse in the charge space coordinates $\left(q_{0}, Q_{0}\right)$,

$$
\frac{q_{0}^{2}}{4 a(0) \kappa_{r}^{2}}+\frac{Q_{0}^{2}}{4 a(0) \kappa_{n}^{2}}=1,
$$

the axes of the ellipse are $2 \sqrt{a(0)} \kappa_{r}$ and $2 \sqrt{a(0)} \kappa_{n}$. Equation (37) contains as a particular case equation (35). A new result for such ellipsoidal polarizations (compared with a similar solution from [10]) is that the parameters of the ellipse (the Kaluza-Klein charges) are modified by an additional solitonic dependence. This describes $3 \mathrm{D}$ solitonic waves in the charge space induced by the 5D vacuum gravitational interactions.

\section{WARPED WORMHOLES IN VARIOUS NON-SPHERICAL BACKGROUNDS}

The locally anisotropic wormhole/flux tube solutions presented in the previous sections are anisotropic deformations from a spherical 3D hypersurface background. These solutions can be generalized to other rotational hypersurface geometry backgrounds. In ref. [10] we gave the explicit forms for such generalized solutions and analyzed their basic properties. The aim of this section is to show that these anisotropic wormhole solutions with ellipsoidal, cylindrical, bipolar and toroidal backgrounds can be solitonically deformed and have a warp factor. The notation and metric relations for the 3D Euclidean rotational hypersurfaces that we use will be those of ref. [22]. In the appendix we outline the necessary results for 3D rotation hypersurfaces and explain the notation (see also refs. [四]). 
In order to construct wormholes which exhibit the various 3D geometries cataloged in the appendix, we will associate respectively one of the ansatz functions of the wormhole solutions with the metric component, $g_{s s}\left(x^{2}, x^{3}\right)$, from (A3), (A6), (A10) and (A12). For the solutions with anisotropic dependence on the extra dimension coordinate this is accomplished by letting $h_{4}=g_{s s}\left(x^{2}, x^{3}\right)$ and then solitonically deforming $h_{4} \rightarrow \widehat{h}_{4}$ and $h_{5} \rightarrow \widehat{h}_{5}$. In each case $h_{4,5}$ is multiplied by the corresponding gravitational polarizations, $\eta_{4,5}$, so as to give wormhole/flux tube configurations of the form (30) and (33), or their modifications presented in section IV. In the previous sections the coordinates were parametrized as $x^{2}=r, x^{3}=\theta$ and $y^{5}=\varphi$. In this section the set $\left(x^{2}, x^{3}, y^{5}\right)$ are spatial coordinates of a $3 \mathrm{D}$ rotation hypersurface. The time like and extra dimensional coordinates are the same as in the previous sections, i.e. $x^{1}=t$ and $y^{4}=\chi$.

Straightforward calculations show that for the five 3D geometries given in the appendix, the metrics defined by (30) or (33) are generalized to a new class of exact solutions of the $5 \mathrm{D}$ vacuum Einstein equations with the coordinates given by

$$
\begin{aligned}
& x^{k}=\left\{\begin{array}{l}
(t, u, v), 0 \leq u<\infty, 0 \leq v \leq \pi, \cosh u \geq 1, \text { ellipsoid (A1); } \\
(t, u, v), 0 \leq u<\infty, 0 \leq v \leq \pi, \sinh u \geq 0, \text { ellipsoid (A4); } \\
(t, u, v), 0 \leq u<\infty, 0 \leq v \leq \pi, \cosh u \geq 1, \text { cylinder (A7); } \\
(t, \tau, \xi),-\infty<\tau<\infty, 0 \leq \xi<\pi, \text { bipolar (A9); } \\
(t, \tau, \xi), 0 \leq \tau<\infty,-\pi<\xi<\pi, \text { torus (A11); }
\end{array}\right. \\
& y^{4}=s=\chi, \quad y^{5}=p=\left\{\begin{array}{l}
\varphi \in[0,2 \pi), \text { ellipsoids ; bipolar ; torus; } \\
z \in(-\infty, \infty), \text { cylinder; }
\end{array}\right.
\end{aligned}
$$

and the ansatz functions given by

$$
\begin{aligned}
& g_{1}=1, g_{2}=-1, g_{3}=-1, \Omega=e^{-k(t)|\chi|}, \\
& \widehat{h}_{4}=\eta_{4} h_{4}, \quad h_{4}=g_{s s}\left(x^{2}, x^{3}\right)=\left\{\begin{array}{l}
\frac{\sinh ^{2} u \sin ^{2} v}{\sinh ^{2} u+\sin ^{2} v}, \text { ellipsoid (A3); } \\
\frac{\sinh ^{2} u \cos ^{2} v}{\sinh ^{2} u+\cos ^{2} v}, \text { ellipsoid (A6); } \\
\frac{\rho^{-2}(u, v)}{\sinh ^{2} u+\sin ^{2} v}, \text { cylinder (A7); } \\
\sin ^{2} \xi, \text { bipolar (A9); torus (A11); }
\end{array},\right. \\
& \eta_{5}=\left\{\begin{array}{l}
\mu^{[K P, G S]}\left(x^{2}, x^{3}, \chi\right), \text { see }(30) ; \\
{\left[1+\varepsilon_{r} \cos \theta\right]^{-2} \mu^{[K P, G S]}\left(x^{2}, x^{3}, \chi\right)^{2}, \text { see }(34) ;} \\
1 / \kappa_{r}^{2}\left(x^{2}, x^{3}, \chi\right), \text { see }(36) ;
\end{array}\right. \\
& \widehat{h}_{5}=\eta_{5} h_{5}, \quad h_{5}\left(x^{2}, x^{3}, \chi\right)=-r_{0}^{2} \exp \left\{2 \psi\left[\left(x^{2}, x^{3}, \chi\right)\right]\right\} ; \quad \zeta_{1}=\zeta_{1}=|\chi| \partial_{t} \ln |k(t)| ; \\
& r=\widetilde{a}^{\text {(invers) }}\left(x^{2}, x^{3}, \chi\right) \text { from (A1); (A4); (A7); (A9); (A11); } \\
& n_{1}=\omega\left(x^{2}\right), \quad n_{2}=0, \quad n_{3}=n \cos x^{3} \times\left\{\begin{array}{l}
\mu^{[K P, G S]}\left(x^{2}, x^{3}, \chi\right), \text { see }(30) ; \\
\mu^{[K P, G S]}\left(x^{2}, x^{3}, \chi\right), \text { see }(33) ; ; ~ \\
1 / \kappa_{n}\left(x^{2}, x^{3}, \chi\right), \text { see }(37) .
\end{array}\right.
\end{aligned}
$$

where the function $\widehat{h}_{5}$ is a solitonic solution of the KdP or SG equations.

Equations (38) describe warped, anisotropic wormhole / flux tube, solitonic configurations which are defined self-consistently in the various rotational hypersurface backgrounds listed above. As in the case of the spherical background these solutions have an anisotropic deformation with respect to the given hypersurface backgrounds. 


\section{CONCLUSIONS}

Some recent work in modern string theory, extra dimensional gravity and particle physics is based on the idea that our 4D universe is embedded in some higher dimension spacetime, but with movement into the higher dimensions being suppressed by an exponential warp factor [11]. The construction and study of wormhole/flux tube solutions in these RS-type models is of fundamental importance in understanding these theories, especially their nonperturbative aspects. Such solutions are difficult to find, and the solutions which are known usually have a high degree of symmetry. In this paper we have applied the method of anholonomic frames to construct warped, solitonic wormholes and flux tubes in 5D KaluzaKlein theory. These solutions have local anisotropy which would make their study using holonomic frames difficult. This demonstrates the usefulness of the anholonomic frames method in handling anisotropic solutions. Most physical situations do not possess a high degree of symmetry, and so the anholonomic frames method provides a useful mathematical framework for studying these less symmetric configurations.

The main result of our work is that we can construct exact solutions in 5D gravity with a warp factor in the extra dimension coordinate which is induced not from any brane energy-momentum configurations but by a specific type of second order anisotropy of the bulk vacuum gravity.

The second key result is the demonstration that off-diagonal metrics in 5D Kaluza-Klein theory can be parametrized into forms that define new, interesting classes of solutions of Einstein's vacuum equations. These solutions represent wormhole and flux tube configurations which are locally anisotropic. These anisotropic solutions reduce to previously known spherically symmetric wormhole metrics [7-9] in the local isotropic limit. These anisotropic solutions also extend the idea of Salam, Strathee and Percacci [6] that including off-diagonal components in higher dimensional metrics gives rise to gauge fields and charges. Not only do we find "electric" and "magnetic" charges for our solutions, but the anisotropies in the $5^{\text {th }}$ coordinate $(\chi)$ and/or in the angular coordinate $(\varphi)$ give a gravitational scaling or running of these Kaluza-Klein charges. Such a gravitational scaling of charges could provide an experimental signature for the presence of extra dimensions (i.e. if some charge were observed to exhibit a running which was not in agreement with that given by $4 \mathrm{D}$ quantum field theory this could be evidence for a gravitational running of the charge).

In connection to the status of off-diagonal metrics in 5D gravity we note that in refs. [12] it was shown that the class of metrics (1) satisfying vacuum Einstein equations (6) -(10) contains as a particular case solutions where the Schwarzschild potential $\Phi=-M /\left(M_{\mathrm{p}}^{2} r\right)$, ( $M_{\mathrm{p}}$ is the effective Planck mass on the brane) is modified to

$$
\Phi=-\frac{M \sigma_{m}}{M_{\mathrm{p}}^{2} r}+\frac{Q \sigma_{q}}{2 r^{2}},
$$

where the "tidal charge" parameter $Q$ may be positive or negative. This points to the possibility of anisotropically modifying Newton's law via an effective anisotropic masses $M \sigma_{m}(t, r, \theta)$, and with an effective gravitational electric-like charge $Q \sigma_{q}(t, r, \theta)$. For diagonal metrics the effective polarizations are $\sigma_{m}=\sigma_{q}=1$. The off-diagonal metrics and

anholonomic frames in extra dimensional vacuum gravity can be used to give non-trivial reductions and trappings to lower dimensional models of wormhole and black hole physics. 
This can result in anisotropic polarizations and scaling of the parameters of the solution, as well as modifications of Newtonian gravity.

In the first part of this paper the warped anisotropic solutions were constructed as deformations from a spherical background. We considered 3D solitonic deformations (KadomtsevPetviashvili and sine-Gordon equations). In the final section of this paper we showed that it is possible to construct a large variety of such warped anisotropic solutions as solitonic deformations from various background geometries: elliptic (elongated and flattened), cylindrical, toroidal and bipolar.

\section{ACKNOWLEDGMENTS}

D.S. would like to thank V. Dzhunushaliev for discussions related to this work. S. V. work is supported both by a 2000-2001 California State University Legislative Award and a NATO/Portugal fellowship grant at the Technical University of Lisabon.

\section{APPENDIX A: 3D ROTATION HYPERSURFACES}

In this appendix we collect together the basic 3D geometric results [1,22] used in section V.

\section{Elongated rotation ellipsoid hypersurfaces}

An elongated rotation ellipsoid hypersurface (a 3D e-ellipsoid) is given by the formula

$$
\frac{x^{2}+y^{2}}{\sigma^{2}-1}+\frac{z^{2}}{\sigma^{2}}=\widetilde{a}^{2}(r)
$$

where $\sigma \geq 1$, and $x, y, z$ are the usual Cartesian coordinates. $\widetilde{a}(r)$ is similar to the radius in the spherical symmetric case. The 3D, ellipsoidal coordinate system is defined

$$
x=\widetilde{a} \sinh u \sin v \cos s, \quad y=\widetilde{a} \sinh u \sin v \sin s, \quad z=\widetilde{a} \cosh u \cos v,
$$

where $\sigma=\cosh u$ and $0 \leq u<\infty, 0 \leq v \leq \pi, 0 \leq s<2 \pi$. The hypersurface metric is

$$
g_{u u}=g_{v v}=\widetilde{a}^{2}\left(\sinh ^{2} u+\sin ^{2} v\right), \quad \widetilde{g}_{s s}=\widetilde{a}^{2} \sinh ^{2} u \sin ^{2} v .
$$

It will be more useful to consider a conformally transformed metric, where the components in equation (A2) are multiplied by the conformal factor $\widetilde{a}^{-2}\left(\sinh ^{2} u+\sin ^{2} v\right)^{-1}$, giving

$$
\begin{aligned}
d s_{(3 e)}^{2} & =d u^{2}+d v^{2}+g_{s s}(u, v) d s^{2} \\
g_{s s}(u, v) & =\sinh ^{2} u \sin ^{2} v /\left(\sinh ^{2} u+\sin ^{2} v\right) .
\end{aligned}
$$




\section{Flattened rotation ellipsoid hypersurfaces}

In a similar fashion we consider the hypersurface equation for a flattened rotation ellipsoid (a 3D f-ellipsoid),

$$
\frac{x^{2}+y^{2}}{1+\sigma^{2}}+\frac{z^{2}}{\sigma^{2}}=\widetilde{a}^{2}(r),
$$

here $\sigma \geq 0$ and $\sigma=\sinh u$. In this case the 3D coordinate system is defined as

$$
x=\tilde{a} \cosh u \sin v \cos s, \quad y=\widetilde{a} \cosh u \sin v \sin s, \quad z=\widetilde{a} \sinh u \cos v,
$$

where $0 \leq u<\infty, 0 \leq v \leq \pi, 0 \leq s<2 \pi$. The hypersurface metric is

$$
g_{u u}=g_{v v}=\tilde{a}^{2}\left(\sinh ^{2} u+\cos ^{2} v\right) \quad g_{\varphi \varphi}=\widetilde{a}^{2} \sinh ^{2} u \cos ^{2} v
$$

Again we consider a conformally transformed version of this metric

$$
\begin{aligned}
d s_{(3 f)}^{3} & =d u^{2}+d v^{2}+g_{s s}(u, v) d s^{2} \\
g_{s s}(u, v) & =\sinh ^{2} u \cos ^{2} v /\left(\sinh ^{2} u+\cos ^{2} v\right) .
\end{aligned}
$$

\section{Ellipsoidal cylindrical hypersurfaces}

The formula for an ellipsoidal cylindrical hypersurface is

$$
\frac{x^{2}}{\sigma^{2}}+\frac{y^{2}}{\sigma^{2}-1}=\rho^{2}, z=s,
$$

where $\sigma \geq 1$. The $3 \mathrm{D}$ radial coordinate is given as $\widetilde{a}^{2}=\rho^{2}+s^{2}$. The $3 \mathrm{D}$ coordinate system is defined

$$
x=\rho \cosh u \cos v, \quad y=\rho \sinh u \sin v, \quad z=s,
$$

where $\sigma=\cosh u$ and $0 \leq u<\infty, 0 \leq v \leq \pi$. Using the expressions for $x, y$ and equation (A7) we can make the change $\rho(x, y) \rightarrow \rho(u, v)$. The hypersurface metric is

$$
g_{u u}=g_{v v}=\rho^{2}(u, v)\left(\sinh ^{2} u+\sin ^{2} v\right), \quad g_{s s}=1
$$

we will again consider a conformally transformed version of this metric

$$
\begin{aligned}
d s_{(3 c)}^{2} & =d u^{2}+d v^{2}+g_{s s}(u, v, \rho(u, v)) d s^{2} \\
g_{s s}(u, v) & =1 / \rho^{2}(u, v)\left(\sinh ^{2} u+\sin ^{2} v\right) .
\end{aligned}
$$




\section{Bipolar coordinates}

Now we consider a bipolar hypersurface given by the formula

$$
\left(\sqrt{x^{2}+y^{2}}-\frac{\widetilde{a}(r)}{\tan \xi}\right)^{2}+z^{2}=\frac{\widetilde{a}^{2}(r)}{\sin ^{2} \xi},
$$

which describes a hypersurface obtained by rotating the circles

$$
\left(y-\frac{\widetilde{a}(r)}{\tan \xi}\right)^{2}+z^{2}=\frac{\widetilde{a}^{2}(r)}{\sin ^{2} \xi}
$$

around the $z$ axis; because $|\tan \xi|^{-1}<|\sin \xi|^{-1}$, the circles intersect the $z$ axis. The relationship between the Cartesian coordinates and the bipolar coordinates is

$$
x=\frac{\widetilde{a}(r) \sin \xi \cos s}{\cosh \tau-\cos \xi}, \quad y=\frac{\widetilde{a}(r) \sin \xi \sin s}{\cosh \tau-\cos \xi}, \quad z=\frac{\widetilde{a}(r) \sinh \tau}{\cosh \tau-\cos \xi},
$$

where $-\infty<\tau<\infty, 0 \leq \xi<\pi, 0 \leq s<2 \pi$. The hypersurface metric is

$$
g_{\tau \tau}=g_{\xi \xi}=\frac{\widetilde{a}^{2}(r)}{(\cosh \tau-\cos \xi)^{2}}, \quad g_{s s}=\frac{\widetilde{a}^{2}(r) \sin ^{2} \xi}{(\cosh \tau-\cos \xi)^{2}},
$$

which, after multiplication by the conformal factor $(\cosh \tau-\cos \sigma)^{2} / \rho^{2}$ becomes

$$
d s_{(3 b)}^{2}=d \tau^{2}+d \xi^{2}+g_{s s}(\xi) d s^{2}, \quad g_{s s}(\xi)=\sin ^{2} \xi
$$

\section{Toroidal coordinates}

Now we consider a toroidal hypersurface with nontrivial topology given by the formula

$$
\left(\sqrt{x^{2}+y^{2}}-\widetilde{a}(r)(\operatorname{coth} \xi)\right)^{2}+z^{2}=\frac{\widetilde{a}^{2}(r)}{\sinh ^{2} \xi}
$$

the relationship to the Cartesian coordinates is given by

$$
x=\frac{\widetilde{a}(r) \sinh \tau \cos s}{\cosh \tau-\cos \xi}, \quad y=\frac{\widetilde{a}(r) \sin \xi \sin s}{\cosh \tau-\cos \xi}, \quad z=\frac{\widetilde{a}(r) \sinh \xi}{\cosh \tau-\cos \xi},
$$

where $-\pi<\xi<\pi, 0 \leq \tau<\infty, 0 \leq s<2 \pi$. The hypersurface metric is

$$
g_{\sigma \sigma}=g_{\tau \tau}=\frac{\tilde{a}^{2}(r)}{(\cosh \tau-\cos \xi)^{2}}, \quad g_{s s}=\frac{\widetilde{a}^{2}(r) \sin ^{2} \xi}{(\cosh \tau-\cos \xi)^{2}}
$$

After multiplication by the conformal factor $(\cosh \tau-\cos \sigma)^{2} / \widetilde{a}^{2}(r)$ this takes the same form as (A10)

$$
d s_{(3 t)}^{3}=d \tau^{2}+d \xi^{2}+g_{s s}(\xi) d s^{2}, \quad g_{s s}(\xi)=\sin ^{2} \xi
$$

Although this looks identical to the metric in (A10) the coordinates $(\tau, \xi, s)$ have different meanings in each case. This can be seen by the different ranges for the two cases. 


\section{REFERENCES}

[1] S. Vacaru, JHEP 04: 009 (2001); Ann. Phys. (NY) 290, 83 (2001); S. Vacaru and F. C. Popa, Class. Quant. Grav. 18, 1 (2001).

[2] S. Vacaru, Ann. Phys. (NY) 256, 39 (1997); Nucl. Phys. B434, 590 (1997); J. Math. Phys. 37, 508 (1996); JHEP 09 : 011 (1998); Phys. Lett. B 498, 74 (2001).

[3] S. Vacaru, D. Singleton, V. Botan and D. Dotenco, Phys. Lett. B 519, 249 (2001).

[4] S. Vacaru, A New Metod of Constructing Black Hole Solutions in Einstein and 5D Gravity, hep-th/0110250.

[5] M. S. Morris and K. S. Thorne, Am. J. Phys., 56, 395 (1988); S. Giddings and A. Strominger, Nucl.Phys., B306, 890 (1988); K. A. Bronnikov and J. C. Fabris, Grav and Cosmol. 3, 67 (1997); M. Rainer and A. Zhuk, Phys.Rev., D54, 6186 (1996); M. Visser, Lorentzian Wormholes: from Einstein to Hawking, (AIP, New York, 1995).

[6] A. Salam and J. Strathdee, Ann. Phys. (NY) 141, 316 (1982); R. Percacci, J. Math. Phys. 24, 807 (1983).

[7] A. Chodos and S. Detweiler, Gen. Rel. Grav. 14, 879 (1982); G. Clément, Gen. Rel. Grav. 16, 131 (1984).

[8] V. D. Dzhunushaliev, Izv. Vuzov, ser. Fizika, 78, N6 (1993)( in Russian); Grav. and Cosmol. 3, 240 (1997); V. D. Dzhunushaliev, Gen. Rel. Grav. 30, 583 (1998); V. D. Dzhunushaliev, Mod. Phys. Lett. A 13, 2179 (1998); V. D. Dzhunushaliev and D. Singleton, Class. Quant. Grav. 16, 973 (1999).

[9] V. D. Dzhunushaliev and D. Singleton, Phys. Rev., D59, 064018 (1999).

[10] S. Vacaru and D. Singleton, Ellipsoidal, Cylindrical, Bipolar and Toroidal Wormholes, hep-th/0110272

[11] L. Randall and R. Sundrum, Phys. Rev. Lett. 833370 (1999); Phys. Rev. Lett. 834690 (1999).

[12] S. Vacaru, Off-Diagonal 5D Metrics and Mass Hierarchies with Anisotropies and Running Constants, hep-ph/0106268; S. Vacaru and E. Gaburov, Anisotropic Black Holes in Einstein and Brane Gravity, hep-th/ 0108065; S. Vacaru and D. Gontsa, Off-Diagonal Metrics and Anisotropic Brane Inflation, hep-th/0109114.

[13] Nima Arkani-Hamed, S. Dimopoulos, N. Kaloper and J. March-Russell, Nucl. Phys. D567, 189 (2000); C. Csaki, M. Graesser, J. Terning, Phys. Lett. B456, 16 (1999); J. Cline, Phys. Rev., D61, 023513 (2000); E. Halyo, JHEP 9909 : 012 (1999); E. Flanagnan, S. H. Henry Tye and Ira Wasserman, Phys. Rev., D62, 024011 (2000); C. Csaki, M. Graesser, L. Randall and J. Terning, Phys. Rev., D62, 045015 (2000); W. D. Goldberger, M. B. Wise, Phys. Lett. B475, 275 (1999); C. Charmousis, R. Gregory and V. Rubakov, Phys. Rev., D62, 067505 (2000).

[14] E. Kamke, Differential Cleichungen. Losungsmethoden und Lonsungen: I. Gewohnliche Differentialgleichungen (Leipzig, 1959).

[15] B. B. Kadomtsev and V. I. Petviashvili, Dokl. Akad. Nauk SSSR, 192, 753 (1970).

[16] V. S. Dryuma, Pis'ma JETP, 19, 753 (1974).

[17] V. E. Zakharov and A. B. Shabat, Funk. Analiz i Ego Prilojenia [in Russian] 8, 43 (1974).

[18] B. Harrison, Phys. Rev. Lett., 41, 1197 (1978).

[19] G. Liebbrandt, Phys. Rev. Lett. 41, 435 (1978).

[20] G. B. Whitham, J. Phys. A. Math., 12, L1 (1979). 
[21] V. A. Belinski and V. E. Zakharov, JETP. 75, 1953 (1978).

[22] G. A. Korn and T. M. Korn, Mathematical Handbook (McGraw-Hill Book Company, 1968). 